PROCEEDINGS OF THE

AMERICAN MATHEMATICAL SOCIETY

Volume 135, Number 10, October 2007, Pages 3041-3047

S 0002-9939(07)08905-8

Article electronically published on May 10, 2007

\title{
ON THE NUMBER OF CERTAIN GALOIS EXTENSIONS OF LOCAL FIELDS
}

\author{
DA-SHENG WEI AND CHUN-GANG JI
}

(Communicated by Ken Ono)

\begin{abstract}
In this paper, we will calculate the number of Galois extensions of local fields with Galois group $A_{n}$ or $S_{n}$.
\end{abstract}

\section{INTRODUCTION}

Let $p$ be a prime, $F$ a finite extension of $p$-adic field $\mathbb{Q}_{p}$ with $\left[F: \mathbb{Q}_{p}\right]=m$. Let $k$ be its residue field with $[k: \mathbb{Z} / p \mathbb{Z}]=f$. It's easy to see that $f \mid m$. Let $\pi$ be a uniformizer of $F$ and $e$ the absolute ramification index of $F / Q_{p}$. Then $m=e f$. In this paper let $\mu_{l}$ denote the set of $l$-th roots of unity. All notations are standard if not explained.

Since the number of the extensions of local fields with a given degree inside the fixed algebraic closure is finite, see [2], one can ask for a formula that gives the number of extensions of a given degree. Krasner [1] gave such a formula, and Serre [6] also computed the number of extensions using a different method. Pauli and Roblot [3] gave the third proof for that formula. Similarly one can also ask for a formula that gives the number of Galois extensions of a given degree. In particular, it is possible to ask for a formula that gives the number of the Galois extensions with the prescribed finite Galois group $G$. We denote this number by $\nu(F, G)$. If $G$ is a $p$-group with $\mu_{p} \nsubseteq F$, Šafarevič [4] gave an explicit formula for the number of the $G$-extensions over $F$ :

$$
\nu(F, G)=\frac{1}{|A u t(G)|}\left(\frac{|G|}{p^{d}}\right)^{m+1} \prod_{i=1}^{d-1}\left(p^{m+1}-p^{i}\right),
$$

where $d$ is the minimal number of generators of $G$. If $G$ is a $p$-group, and $\mu_{p} \subset F$, Yamagishi [7] obtained a formula for $\nu(F, G)$.

In this paper, we will calculate the number of $S_{n}$-extensions and $A_{n}$-extensions over $F$, where $S_{n}$ is the $n$-th symmetric group and $A_{n}$ is the $n$-th alternating group.

The cases for $n \geq 5$ that are quickly dismissed as $S_{n}$ and $A_{n}$ are not solvable in these cases, and the Galois groups of extensions of local fields are always solvable. So we only need to handle the remaining cases, $n \leq 4$.

Received by the editors June 13, 2006.

2000 Mathematics Subject Classification. Primary 11S15, 11S20.

Key words and phrases. Local fields, Galois closure, ramified extensions.

This work was partially supported by grants \#10171046 and \#10201013 from NNSF of China and Jiangsu planned projects for postdoctoral research funds.

(C)2007 American Mathematical Society 
Theorem 1.1. Let $F$ be a finite extension over $\mathbb{Q}_{p}$ with $\left[F: \mathbb{Q}_{p}\right]=m, \nu(F, G)$ the number of the Galois extensions $K / F$ with $G a l(K / F)=G$.

(1) Suppose the prime $p \neq 3$; then

$$
\nu\left(F, S_{3}\right)= \begin{cases}0 & \text { if } \mu_{3} \subset F, \\ 3 & \text { if } \mu_{3} \not \subset F .\end{cases}
$$

(2) Suppose $p=3$; then

$$
\nu\left(F, S_{3}\right)= \begin{cases}3^{m+1}-3 & \text { if } \mu_{3} \subset F, \\ 3^{m}+\frac{3^{m+1}}{2}-\frac{3}{2} & \text { if } \mu_{3} \not \subset F .\end{cases}
$$

Theorem 1.2. Let $F$ be a finite extension over $\mathbb{Q}_{p}$ with $\left[F: \mathbb{Q}_{p}\right]=m, \nu(F, G)$ the number of the Galois extensions $K / F$ with $G a l(K / F)=G$.

(1) Suppose the prime $p \geqslant 3$; then

$$
\nu\left(F, S_{4}\right)=\nu\left(F, A_{4}\right)=0 .
$$

(2) Suppose $p=2$; then

$$
\begin{gathered}
\nu\left(F, A_{4}\right)= \begin{cases}4\left(2^{2 m}-1\right) / 3 & \text { if } \mu_{3} \subset F, \\
\left(2^{2 m}-1\right) / 3 & \text { if } \mu_{3} \not \subset F ;\end{cases} \\
\nu\left(F, S_{4}\right)= \begin{cases}0 & \text { if } \mu_{3} \subset F, \\
2^{2 m+1}-1 & \text { if } \mu_{4} \nsubseteq F \text { and } m \text { is even and } f=1, \\
2^{2 m}-1 & \text { otherwise. }\end{cases}
\end{gathered}
$$

\section{Some Lemmas}

The number of $S_{2}$-extensions and $A_{3}$-extensions of local fields is specified by wellknown results of local class field theory. So we only need to calculate the number of Galois extensions over $F$ with Galois group $S_{3}, S_{4}$ and $A_{4}$. The following lemma plays an important role in our calculation.

Lemma 1. Let $K$ be a Galois extension over $F$ with the Galois group $G$. For any subgroup $A$ of $G$, let $F_{A}$ be the field fixed by $A$. Then the Galois closure cl $\left(F_{A}\right)$ of $F_{A}$ is a subfield of $K$ and $\operatorname{Gal}\left(K / \operatorname{cl}\left(F_{A}\right)\right)=\bigcap_{g \in G} g A g^{-1}$.

We can get some Galois extensions from some non-Galois extensions by taking their Galois closure. For example, if $\mathrm{G}=S_{3}, D_{8}, A_{4}$ and $S_{4}$, we can choose $A$ to be isomorphic to $\mathbb{Z} / 2 \mathbb{Z}, \mathbb{Z} / 2 \mathbb{Z}, \mathbb{Z} / 3 \mathbb{Z}$ and $S_{3}$, which is the non-normal subgroup of $G$ respectively, and where $D_{8}$ is the 2-sylow subgroup of $S_{4}$. By the above lemma, the Galois extensions of $F$ with Galois group $S_{3}$ can be gotten by the Galois closure of extensions of degree 3 of $F$, and the Galois extensions of $F$ with Galois group $D_{8}$, $A_{4}$ and $S_{4}$ can be gotten by the Galois closure of extensions of degree 4 of $F$.

Let $M(n)$ denote the set of all extensions of degree $n$ of $F$. Let $A b(n)$ denote the set of abelian extensions of degree $n$ of $F$. Also, let $M(G)$ denote the set of Galois extensions of $F$ with the Galois group $G$. Let $K$ be the Galois closure of an extension of degree $n$ of $F$. The Galois group $\operatorname{Gal}(K / F)$ is a subgroup of $S_{n}$. Obviously the order of $\operatorname{Gal}(K / F)$ must be divided by $n$. So there are the following two maps:

$$
f: \quad M(3) \rightarrow A b(3) \cup M\left(S_{3}\right)
$$


and

$$
g: \quad M(4) \rightarrow A b(4) \cup M\left(D_{8}\right) \cup M\left(A_{4}\right) \cup M\left(S_{4}\right)
$$

by

$$
L \rightarrow \operatorname{cl}(L) .
$$

The two maps are surjective. Any inverse image $L$ of an element $K$ in $M(G)$ is a subfield of $K$, and $L$ is not a Galois extension of $F$ if $G$ is not an abelian group. In these cases, the Galois group $\operatorname{Gal}(K / L)$ is not a normal subgroup of $G$. For $G=S_{3}, D_{8}, A_{4}$ and $S_{4}$, we consider respectively the number of non-normal subgroups isomorphic to $\mathbb{Z} / 2 \mathbb{Z}, \mathbb{Z} / 2 \mathbb{Z}, \mathbb{Z} / 3 \mathbb{Z}$ and $S_{3}$. The subgroups are non-normal except there is an order-2 normal subgroup in $D_{8}$. So the number of inverse images of any element in $M\left(S_{3}\right), M\left(D_{8}\right), M\left(A_{4}\right)$ and $M\left(S_{4}\right)$ are 3,4,4,4 respectively. Let $|S|$ denote cardinality of a finite set $S$. Let $\nu(F, G)$ denote the number of $M(G)$. So there is the following result.

\section{Lemma 2.}

$$
\begin{gathered}
|M(3)|=|A b(3)|+3 \nu\left(F, S_{3}\right), \\
|M(4)|=|A b(4)|+4 \nu\left(F, D_{8}\right)+4 \nu\left(F, A_{4}\right)+4 \nu\left(F, S_{4}\right) .
\end{gathered}
$$

\section{The Proof of Theorem 1.1}

In the following, we denote $m=\left[F: \mathbb{Q}_{p}\right]$, and $e$ is the absolute ramification index of $F$ and $q=p^{f}$ is the number of elements of the residue field of $F$.

Proof. (1) For $p \neq 3$,

$$
M(3)=\{K \mid[K: F]=3, K \text { is a tamely ramified extension of } F\} .
$$

(i) If $3^{\text {rd }}$ roots of unity are contained in $F$, then $M(3)=A b(3)$. By Lemma 2

$$
\nu\left(F, S_{3}\right)=0 .
$$

(ii) If $3^{\text {rd }}$ roots of unity are not contained in $F$, then $|M(3)|=10,|A b(3)|=1$. By Lemma 2

$$
\nu\left(F, S_{3}\right)=3 .
$$

(2) For $p=3$, by Krasner's theorem [3,

$$
|M(3)|=3 q^{e}+6(q-1)\left(\sum_{a=0}^{e-1} q^{a}\right)+1=9 q^{e}-5 .
$$

Suppose $\mu_{3} \not \subset F$; then

$$
|A b(3)|=\frac{1}{2}\left(\frac{3}{3}\right)^{m+1}\left(3^{m+1}-1\right)=\frac{3^{m+1}-1}{2}=\frac{3 q^{e}-1}{2} .
$$

Suppose $\mu_{3} \subset F$; then

$$
|A b(3)|=4 .
$$

By Lemma 2

$$
\nu\left(F, S_{3}\right)= \begin{cases}\frac{5 q^{e}-3}{2} & \text { if } \mu_{3} \not \subset F \\ 3 q^{e}-3 & \text { if } \mu_{3} \subset F .\end{cases}
$$




\section{The Proof of Theorem 1.2}

First we give some propositions.

Proposition 4.1. Let the prime $p \geqslant 3$. Then

$$
\nu\left(F, S_{4}\right)=\nu\left(F, A_{4}\right)=0 \text {. }
$$

Proof. Suppose $K$ is a Galois extension over $F$ with Galois group $S_{4}$. There must exist intermediate fields $F^{t r}$ and $F^{u r}$ such that $\operatorname{Gal}\left(K / F^{t r}\right)$ is a $p$-group, and $\operatorname{Gal}\left(F^{t r} / F^{u r}\right)$ and $\operatorname{Gal}\left(F^{u r} / F\right)$ are cyclic groups. By Galois theory, there is a $p$ group $S^{\prime}$ which is a normal subgroup of $S_{4}$. Since $p \geqslant 3, S^{\prime}$ must be (1). Since $S_{4}$ does not have a cyclic normal subgroup $S$ such that $S_{4} / S$ is also cyclic, this is a contradiction.

Similarly we get $\nu\left(F, A_{4}\right)=0$.

Proposition 4.2. Let $p=2$. Then

$$
\nu\left(F, A_{4}\right)= \begin{cases}4\left(2^{2 m}-1\right) / 3 & \text { if } \mu_{3} \subset F, \\ \left(2^{2 m}-1\right) / 3 & \text { if } \mu_{3} \not \subset F .\end{cases}
$$

Proof. Let $K$ be an $A_{4}$-extension over $F$. Since $K_{4}$ is a normal subgroup of $A_{4}$, there exists a (unique) Galois subfield $F^{\prime}$ of degree 3 over $F$, where $K_{4} \cong \mathbb{Z} / 2 \mathbb{Z} \times \mathbb{Z} / 2 \mathbb{Z}$. By [2],

$$
\begin{aligned}
\left|F^{* *} /\left(F^{*}\right)^{2}\right| & =4 q^{3 e}, \\
\left|F^{*} /\left(F^{*}\right)^{2}\right| & =4 q^{e} .
\end{aligned}
$$

It is clear that the natural map of $F^{*} /\left(F^{*}\right)^{2} \rightarrow F^{\prime *} /\left(F^{\prime *}\right)^{2}$ is an injection since $\left[F^{\prime}: F\right]=3$. We consider the action on $F^{\prime *} /\left(F^{\prime *}\right)^{2}$ of the $\operatorname{Galois} \operatorname{group} \operatorname{Gal}\left(F^{\prime} / F\right)$.

(1) Denote $G^{\prime}=\operatorname{Gal}\left(F^{\prime} / F\right)$. Then the following result holds:

$$
\left(F^{*} /\left(F^{*}\right)^{2}\right)^{G^{\prime}} \cong F^{*} /\left(F^{*}\right)^{2} .
$$

As we've already noted injectivity, it remains to show that the natural map is surjective. Let $a \in F^{*} /\left(F^{* *}\right)^{2}$ be a fixed point of $\operatorname{Gal}\left(F^{\prime} / F\right)$ and $a \neq 1$ in $F^{*} /\left(F^{\prime *}\right)^{2}$. Then $F^{\prime}\left(\sqrt{a^{\prime}}\right)$ is a Galois extension over $F$, where $a^{\prime}$ represents a lifting of $a$ in $F^{\prime *}$. There isn't an order-2 normal subgroup in $S_{3}$, so

$$
\operatorname{Gal}\left(F^{\prime}\left(\sqrt{a^{\prime}}\right) / F\right) \cong \mathbb{Z} / 6 \mathbb{Z} .
$$

Let $F^{\prime \prime}$ be the fixed field of the normal subgroup $\mathbb{Z} / 3 \mathbb{Z}$. There exists an element $b \in F^{*} /\left(F^{*}\right)^{2}$ such that

$$
F^{\prime \prime}=F(\sqrt{b})
$$

Then

$$
F^{\prime}\left(\sqrt{a^{\prime}}\right)=F^{\prime}(\sqrt{b})
$$

So $a=b$ in $F^{\prime *} /\left(F^{\prime *}\right)^{2}$.

(2) Let $\sigma$ be a generator of $G^{\prime}$. Assume $x \in F^{\prime *} /\left(F^{* *}\right)^{2}-F^{*} /\left(F^{*}\right)^{2}$; then there are the following two cases:

(i) $N_{F^{\prime} / F}(x)=1$ in $F^{*} /\left(F^{*}\right)^{2}$,

(ii) $N_{F^{\prime} / F}(x) \neq 1$ in $F^{*} /\left(F^{*}\right)^{2}$. 
In (i), the field $F^{\prime}(\sqrt{x}, \sqrt{\sigma x})$ is an $A_{4}$-extension over $F$ since the Galois group of $F^{\prime}(\sqrt{x}, \sqrt{\sigma x}) / F$ isomorphic to $K_{4} \rtimes \mathbb{Z} / 3 \mathbb{Z}$, where $K_{4} \cong \mathbb{Z} / 2 \mathbb{Z} \times \mathbb{Z} / 2 \mathbb{Z}$.

In (ii),

$$
F^{\prime}\left(\sqrt{x}, \sqrt{\sigma x}, \sqrt{\sigma^{2} x}\right)=F^{\prime}\left(\sqrt{x / \sigma(x)}, \sqrt{\sigma x / \sigma^{2} x}, \sqrt{N_{F^{\prime} / F}(x)}\right),
$$

So $F^{\prime}\left(\sqrt{x}, \sqrt{\sigma x}, \sqrt{\sigma^{2} x}\right)$ is an $\left(A_{4} \times \mathbb{Z} / 2 \mathbb{Z}\right)$-extension over $F$. Any $\left(A_{4} \times \mathbb{Z} / 2 \mathbb{Z}\right)$ extension $K$ is generated by an $A_{4}$-extension over $F$ and an extension of degree 2 over $F$. Denote the unique extension of degree 3 by $F^{\prime}$; then there exist $x \in$ $F^{\prime *} /\left(F^{*}\right)^{2}$ and $a \in F^{*} /\left(F^{*}\right)^{2}$ satisfying $x \notin F^{*} /\left(F^{*}\right)^{2}, N_{F^{\prime} / F}(x) \in\left(F^{*}\right)^{2}$ and $a \notin\left(F^{*}\right)^{2}$, such that $K=F^{\prime}(\sqrt{x}, \sqrt{\sigma x}, \sqrt{a})$. It is easy to see that

$$
K=F^{\prime}\left(\sqrt{a x / \sigma x}, \sqrt{a \sigma x / \sigma^{2} x}, \sqrt{a \sigma^{2} x / x}\right)
$$

since $N_{F^{\prime} / F}(x)=x \sigma x \sigma^{2} x=1$ in $F^{*} /\left(F^{*}\right)^{2}$. Then $\sigma x / \sigma^{2} x=x \sigma^{2} x / \sigma^{2} x=x$ in $F^{*} /$ $\left(F^{\prime *}\right)^{2}$. Consider $K$ as an extension over $F^{\prime}$; there exist 7 subfields with order 2 over $F^{\prime}$ which are one-to-one correspondents to $\left\{y, \sigma y, \sigma^{2} y, y \sigma y, \sigma y \sigma^{2} y, y \sigma^{2} y, N_{F^{\prime} / F}(y)\right\}$, where $y=a x / \sigma x$. And $N_{F^{\prime} / F}(y)=a \neq 1$ in $F^{*} /\left(F^{*}\right)^{2}$. The $\operatorname{Gal}\left(F^{\prime} / F\right)$-orbits are $\left\{y, \sigma y, \sigma^{2} y\right\},\left\{y \sigma y, \sigma y \sigma^{2} y, y \sigma^{2} y\right\}$, and $\left\{N_{F^{\prime} / F}(y)\right\}$. So any $\left(A_{4} \times \mathbb{Z} / 2 \mathbb{Z}\right)$-extension over $F$ is in form of (ii) and

$$
\nu\left(F, A_{4} \times \mathbb{Z} / 2 \mathbb{Z}\right)=\nu\left(F, A_{4}\right) \nu(F, \mathbb{Z} / 2 \mathbb{Z}) .
$$

(i) Suppose $\mu_{3} \not \subset F, F^{\prime}$ is the unique unramified extension of degree 3 over $F$, so

$$
\begin{gathered}
3 \nu\left(F, A_{4}\right)+3 \nu\left(F, A_{4} \times \mathbb{Z} / 2 \mathbb{Z}\right)=4 q^{3 e}-4 q^{e}, \\
\nu\left(F, A_{4} \times \mathbb{Z} / 2 \mathbb{Z}\right)=\nu\left(F, A_{4}\right)\left(4 q^{e}-1\right) .
\end{gathered}
$$

Then

$$
\nu\left(F, A_{4}\right)=\left(q^{2 e}-1\right) / 3 .
$$

(ii) Suppose $\mu_{3} \subset F, F^{\prime}$ is the unique unramified extension and 3 totally ramified extensions of degree 3 over $F$, so

$$
\begin{gathered}
3 \nu\left(F, A_{4}\right)+3 \nu\left(F, A_{4} \times \mathbb{Z} / 2 \mathbb{Z}\right)=4\left(4 q^{3 e}-4 q^{e}\right), \\
\nu\left(F, A_{4} \times \mathbb{Z} / 2 \mathbb{Z}\right)=\nu\left(F, A_{4}\right)\left(4 q^{e}-1\right) .
\end{gathered}
$$

Then

$$
\nu\left(F, A_{4}\right)=4\left(q^{2 e}-1\right) / 3 .
$$

Proposition 4.3. Let $p=2$. Then

$$
\nu\left(F, S_{4}\right)= \begin{cases}0 & \text { if } \mu_{3} \subset F, \\ 2^{2 m+1}-1 & \text { if } \mu_{4} \nsubseteq F \text { and } m \text { is even and } f=1, \\ 2^{2 m}-1 & \text { otherwise. }\end{cases}
$$

Proof. By Krasner's theorem [3],

$$
|M(4)|=16 q^{3 e}-4 q^{2 e}-5 .
$$

By the local class field theory and the dual theory of the finite abelian group, the following equation holds:

$$
|A b(4)|=\mid\left\{S: S \text { is the subgroup of order } 4 \text { of } F^{*} /\left(F^{*}\right)^{4}\right\} \mid \text {. }
$$


Let $T_{1}$ be the set consisting of the elements of order $\leqslant 2$ in $F^{*} /\left(F^{*}\right)^{4}$, and let $T_{2}$ be the set consisting of the elements of order 4 in $F^{*} /\left(F^{*}\right)^{4}$. The sequence

$$
0 \rightarrow T_{1} \rightarrow F^{*} /\left(F^{*}\right)^{4} \rightarrow\left(F^{*}\right)^{2} /\left(F^{*}\right)^{4} \rightarrow 0
$$

is exact. The third map is $a \mapsto a^{2}$. So

$$
\left|T_{1}\right|=\left|F^{*} /\left(F^{*}\right)^{4}\right| /\left|\left(F^{*}\right)^{2} /\left(F^{*}\right)^{4}\right| .
$$

Suppose $\mu_{4} \not \subseteq F$; then

$$
\begin{gathered}
\left|T_{1}\right|=4 q^{e}, \\
\left|T_{2}\right|=8 q^{2 e}-4 q^{e} .
\end{gathered}
$$

Then

$$
|A b(4)|=\left|T_{2}\right| / 2+\left(\left|T_{1}\right|-1\right)\left(\left|T_{1}\right|-2\right) / 6=20 q^{2 e} / 3-4 q^{e}+1 / 3 .
$$

Suppose $\mu_{4} \subset F$; then

$$
\begin{gathered}
\left|T_{1}\right|=4 q^{e}, \\
\left|T_{2}\right|=16 q^{2 e}-4 q^{e} .
\end{gathered}
$$

Then

$$
|A b(4)|=\left|T_{2}\right| / 2+\left(\left|T_{1}\right|-1\right)\left(\left|T_{1}\right|-2\right) / 6=32 q^{2 e} / 3-4 q^{e}+1 / 3 .
$$

Since $D_{8}$ is a 2-group, by Theorem 2.2 of [7],

$$
\nu\left(F, D_{8}\right)= \begin{cases}q^{e}\left(q^{e}-1\right)\left(4 q^{e}-1\right) & \text { if } \mu_{4} \subset F \text { or } \mu_{4} \nsubseteq F \text { and } m \text { is even and } f=1, \\ q^{e}\left(2 q^{e}-1\right)^{2} & \text { otherwise. }\end{cases}
$$

By Lemma 2,

(i) If $\mu_{4} \subset F$, then

$\nu\left(F, S_{4}\right)=(|M(4)|-|A b(4)|) / 4-\nu\left(F, A_{4}\right)-\nu\left(F, D_{8}\right)=4\left(q^{2 e}-1\right) / 3-\nu\left(F, A_{4}\right)$.

(ii) If $\mu_{4} \nsubseteq \subseteq F$, and $m$ is odd or $m$ is even and $f \geqslant 2$, then

$\nu\left(F, S_{4}\right)=(|M(4)|-|A b(4)|) / 4-\nu\left(F, A_{4}\right)-\nu\left(F, D_{8}\right)=4\left(q^{2 e}-1\right) / 3-\nu\left(F, A_{4}\right)$.

(iii) If $\mu_{4} \nsubseteq F$ and $m$ is even and $f=1$, then

$$
\nu\left(F, S_{4}\right)=(|M(4)|-|A b(4)|) / 4-\nu\left(F, A_{4}\right)-\nu\left(F, D_{8}\right)=\left(7 q^{2 e}-4\right) / 3-\nu\left(F, A_{4}\right) .
$$

By Proposition 4.2, we have

$$
\nu\left(F, S_{4}\right)= \begin{cases}0 & \text { if } \mu_{3} \subset F, \\ 2 q^{2 e}-1 & \text { if } \mu_{4} \nsubseteq F \text { and } n \text { is even and } f=1, \\ q^{2 e}-1 & \text { otherwise. }\end{cases}
$$

Remark. Since $K_{4}$ is a normal subgroup of $S_{4}$ and $S_{4} / K_{4} \cong S_{3}$, there exists an $S_{3}$-subextension in an $S_{4}$-extension of $F$ by Galois theory. If $\mu_{3} \subset F$ and $p \neq 3$, then $\nu\left(F, S_{3}\right)=0$. So $\nu\left(F, S_{4}\right)=0$. This gives another proof for a case of $\nu\left(F, S_{4}\right)$.

Using these propositions, the proof of Theorem 1.2 is obtained. This completes the proof of Theorem 1.2. 


\section{EXAMPLES}

Example 5.1. Let $F=\mathbb{Q}_{p}$. Then

(1)

$$
\nu\left(\mathbb{Q}_{p}, S_{3}\right)=\left\{\begin{array}{lll}
6 & \text { if } p=3, \\
0 & \text { if } p \equiv 1 \\
3 & \text { if } p \equiv 2 & \bmod 3 \\
&
\end{array}\right.
$$

$$
\nu\left(\mathbb{Q}_{p}, A_{4}\right)= \begin{cases}1 & \text { if } p=2, \\ 0 & \text { if } p>2\end{cases}
$$

$$
\begin{gathered}
\nu\left(\mathbb{Q}_{p}, S_{4}\right)= \begin{cases}3 & \text { if } p=2, \\
0 & \text { if } p>2 .\end{cases} \\
\nu\left(\mathbb{Q}_{p}, S_{n}\right)=\nu\left(\mathbb{Q}_{p}, A_{n}\right)=0 \quad(n \geqslant 5) .
\end{gathered}
$$

\section{REFERENCES}

[1] M. Krasner, Nombre des extensions d'un degré donné d'un corps $p$-adique, les Tendances Géométriques en algébre et Théorie des Nombres, ED. CNRS, Paris, 1966, 143-169. MR0225756 (37:1349)

[2] S. Lang, Algebraic Number Theory, Second edition. GTM, 110. Springer-Verlag, New York, 1994. MR1282723 (95f:11085)

[3] S. Pauli and X. F. Roblot, On the computation of all extensions of a $p$-adic field of a given degree, Math. Comp. 70 (2001), no. 236, 1641-1659. MR1836924(2002e:11166)

[4] I. R. Šafarevič, On p-extensions, Mat. Sb. 20(62)(1947), 351-363; English transl., Amer. Math. Soc. Transl. Ser. 24 (1956), 59-72. MR0020546 (8:560e)

[5] J. -P. Serre, Corps Locaux, Hermann, Paris, 1963. MR0354618 (50:7096)

[6] J. -P. Serre, Une "formule de masse" pour les extensions totalement ramifiées de degré donné d'un corps local, C. R. Acad. Sci. Pairs Sér. A-B 286 (1978), A1031-A1036. MR500361 (80a:12018)

[7] M. Yamagishi, On the number of Galois $p$-extensions of a local field, Proc. Amer. Math. Soc. 123 (1995), 2373-2380. MR.1264832 (95j:11109)

Department of Mathematics, the University of Science and Technology of China, Hefei, People's Republic of China 230026

E-mail address: dshwei@ustc.edu

Department of Mathematics, Nanjing Normal University, Nanjing, People's Republic of China 210097

E-mail address: cgji@njnu.edu.cn 